\title{
Erratum to: Discussion on Failure Mechanism and Strength Criterion of Sandstone Based on Particle Discrete Element Method
}

\author{
Shuguang Zhang ${ }^{\mathrm{a}, \mathrm{b}}$, Lei Chen ${ }^{\oplus a}$, Pingping Lu ${ }^{\mathrm{a}}$, and Wenbo Liu ${ }^{\mathrm{a}}$ \\ ${ }^{a}$ Civil Engineering Institute, Liaoning Technical University, Fuxing 123000, China \\ ${ }^{b}$ Guangxi Key Laboratory of Geotechnical Mechanics and Engineering, Guilin University of Technology, Guilin 541004, China
}

Erratum to: KSCE Journal of Civil Engineering (2021) 25(6):2314-2333

$10.1007 / \mathrm{s} 12205-021-2031-4$

This erratum is published to notify corrections in the author byline. See the corrected version below:

\section{Errata:}

The original version of this article:

\author{
Shuguang Zhang ${ }^{\mathrm{a}, \mathrm{b}}$, Lei Chen ${ }^{\oplus \mathrm{b}}$, Pingping Lu ${ }^{\mathrm{b}}$, and Wenbo Liu \\ ${ }^{a}$ Guangxi Key Laboratory of Geotechnical Mechanics and Engineering, Guilin University of Technology, Guilin 541004, China \\ ${ }^{b}$ Civil Engineering Institute, Liaoning Technical University, Fuxing 123000, China
}

Was altered as:

\section{Shuguang Zhang ${ }^{\mathrm{a}, \mathrm{b}}$, Lei Chen ${ }^{\circledR a}$, Pingping $\mathrm{Lu}^{\mathrm{a}}$, and Wenbo Liu ${ }^{\mathrm{a}}$}

${ }^{b}$ Guangxi Key Laboratory of Geotechnical Mechanics and Engineering, Guilin University of Technology, Guilin 541004, China 